\title{
EXTRA-UTERINE PREGNANCY.
}

\section{By GILBERT BRESCHET, M.D.}

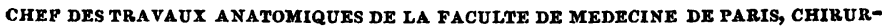
GIEN EN CHEF DE L' HOSPICE DES ENFANS-TROUVES, PROFESSEU R AGREGE A LA FACULTE DE MEdecine, PROFESSEUR PARTICULIER D'ANATOMIE, MEMbRE TITU I.AIRA DE I'ACADEML ROYALE dE MEDECINE, DE LA SOCIETe PHILOMATIQUe, hTG.

Read Feb. 23d, 1824.

$\mathbf{I T}_{\mathrm{T}}$ is agreed by modern anatomists that the product of generation passes from the ovarium into the fallopian tube, before entering the uterus where it is to acquire its full developement. The ovum, however, does not invariably follow this regular course, but may be impeded at some point in its passage; the pregnancy is then termed extrauterine, and of this there are three varieties. In the first variety, graviditas abdominaiis, or ventral pregnancy, the embryo is in the abdominal cavity, and its involucra form adhesions to the viscera and the peritoneum. In the second, graviditas ovariana, ovarian pregnancy, the fotus is developed in the ovarium; and in the third, graviditas tubaria, or tubal pregnancy, the ovum does not pass the tube, but acquires a certain growth, varied by circumstances, in that canal. I am of opinion that a 
fourth species of extra-uterine pregnancy may be admitted, which I shall term, Graviditas in Uteri substantia. Of this, few cases have been recorded, but they are from authorities of weight. It is a case of this description which I have now the honour to submit to the Medical and Chirurgical Society, and it is the first which has been published in France or England.

In this description of extra-uterine pregnancy, the-fœtus is enclosed in the parenchyma of the uterus itself, excepting that a cyst separates it from the substance of the viscus, as is the case in the instance of foreign bodies introduced into organic textures, and as is particularly observed in hydatids and some other Zoophytes. No communication exists between the cavity containing the fotus and the cavities of the abdomen or uterus. I have lately received from Drs. Bellemain and Lartez, an anatomical preparation, illustrative of this species of extra-uterine pregnancy.

Madame B., after experiencing the usual disagreeable feelings which accompany pregnancy, was attacked on the 10th of June, 1823, with urgent vomiting and violent pain in the hypogastric region, extending to the rectum. She suffered also from frequent fits of fainting, and from a general sense of debility. The countenance and lips were pallid, and the pulse small. Rest was recommended, and an antispasmodic mixture prescribed. 
The abdomen, which had not at first indicated pain on pressure, now became exquisitely tender. On examination per vaginam, a well defined enlargement of the uterus was discovered without any developement of the cervix. The patient stated that she was three months gone with child. Twenty leeches were applied to the abdomen, and a warm bath was directed to be used. All the bad symptoms increased, and on the 11 th the patient died. Her medical attendants attributed her death to a sub-acute inflammation of the peritoneum, or to the rupture of a great blood-vessel in the abdominal or pelvic cavity.

On opening the abdomen, a considerable effusion of blood, partly liquid and partly coagulated, was discovered in the cavity of the pelvis. The uterus, although increased in size, had not risen above the brim of the pelvis, and at its fundus, towards the left side, was discovered a rupture which included the peritoneum and the cellular substance of the viscus, without establishing any communication between the cavities of the abdomen and the uterus, as was at first supposed. Through this rupture of the uterus, a foetus included within its membranes protruded. The drawing which accompanies this memoir, represents the preparation in question, in which the uterus, from five to six inches long, and four broad, presents a cavity of sufficient size to contain a hen's egg. In it were found some mucosity or albumen, and a mem- 
brane, which was certainly the membrana decidua of Hunter. The parietes of the uterus were sixteen to eighteen lines in thickness; consequently, this organ was much larger than when unimpregnated. The ovaries were healthy; the right fallopian tube was obliterated in its upper half, and the left tube in its whole extent. The most careful dissection could not detect its canal, nor could any liquid be forced through it by the syringe; but the lower orifice of the tube was preserved in the superior and left angle of the cavity of the uterus, and this cavity was much below the cavity containing the foetus. The cyst containing the foetus, was formed in the substance of the fundus of the uterus, above the insertion of the left fallopian tube. It was not lined by any membrane. In size it equalled an egg, and on its surface, which was very irregular, we observed the orifices of numerous vessels or uterine sinuses; and in these sinúses were seen some vascular threads or penicilli, forming the rudiments of the placenta. The partition dividing this cavity from the uterus, was half an inch thick, but the upper portion of its parietes, i. e. the part which corresponds with the fundus of the uterus, and on which the rupture took place, was only two lines thick. It was, however, evidently formed of two distinct parts, namely, the peritoneum, and the substance of the uterus. I make this remark, because some Physiologists.* are of

* Carl. Gust. Carus, zur lehre von Schwangenschaft und 
opinion that in this species of extra-uterine pregnancy the ovum escapes, and lodges between the peritoneum and the uterus.

We observed the formation of numerous vessels in the substance of the uterus, immediately surrounding the cyst, indicating great activity of circulation ; no opening or communication existed between the cavity containing the foetus and the cavity of the uterus, properly so called.

The foetus, with its involucra, was several inches long. The external surface of the chorion exhibited at a great many points, instead of a regular placenta, cotyledons, as it were, attached to the chorion by long pedicles. This arrangement resembles that of the ovum in ruminating animals, * and one of these bundles of vascular penicilli, a species of cotyledon, was moreover employed in connecting the ovum with the mother.

I am indebted to the friendship of Dr. Albers, of Bremen, for the knowledge of some cases similar to the one I have described. In a letter I received from him a short time before his death, he informed me that he was called to a lady, three months advanced

Geburt physiologische, pathologische und therapeutische Abhandlungen, \&c. Leipsig, 1822.

* Ueber das Gebaerorgan der Menschen und der Saugthiere in Schwangern und nicht Schwangern Zustande von Dr. Joh. C. Gotfried. Jorg. Leipsig. 1808. 
in pregnancy, who, aftergetting upin perfect health, was suddenly seized in the course of the morning with very violent pains in the abdomen. At his first visit, Albers suspected that the pregnancy was extra-uterine, and that the symptoms resulted from the rupture of the fallopian tube, or of a cyst, including a foetus. A few hours afterwards, he found his patient pale and cold as a corpse; the lips of a violet blue; the pulse frequent and very small. She was lying on her side in a state of quiet, and only complained when questioned as to her feelings. She then stated that she suffered violent pains of the abdomen, chiefly affecting the umbilical region; these pains continued till six in the evening, when they ceased: the patient, however, was conscious of her approaching end, and she expired a few minutes afterwards. Albers assured me that at his second visit, he was perfectly certain of the nature of the disease, and referred to his learned friend Dr. Olbers, in confirmation of this assurance.

On opening the body twenty-four hours aiter death, in the presence of Drs. Schmidt, father and son, he discovered a rupture of the uterus, through which a very small foetus had escaped; and, in the letter in which he related the case to me, he mentioned that the ovum had developed itself in an extraordinary manner in the substance of the womb, and that he could discover no communication between the cyst and the uterine cavity. I receiv- 
ed no farther particulars of this case, as it was Dr. Albers' intention to transmit it to the Medical and Chirurgical Society of London. The death of my lamented friend however frustrated his intention.*

There are only two cases on record, similar to those I have now described, which I believe are not much known.

The first is to be found in the first volume of the memoirs of the Josephine Academy of Vienna, and is related by Professor G. Schmittt.-A woman aged 28, had been pregnant five times; and the four first deliveries had been effected in the most favourable manner, but in the succeeding pregnancy she only went to the fifth month. This woman's health had been invariably good, with the exception of slight hysteria with which she was attacked in her last gestation. About a month be-

* In a letter which I have just received from Dr. Roget, Secretary to the Medical and Chirurgical Society, I am informed that no communication has been received from Dr. Albers on the subject alluded to. Dr. Albers must have examined two cases of this nature, for it is mentioned in Dr. Carus' work that Albers a short time before his death, purchased for a considerable sum an anatomical preparation in which the fotus was in the substance of the uterus, and that he intended to forward a description of it to the Medical and Chirurgical Society of London.

$\uparrow$ Memoires et Observat. de l'Acad. Medico-Chir. de Vienne. Vienne 1801. 
fore her death, on returning from a short journey, she was seized with a sensation of pain and weight in the stomach. 'This dull pain continued for ten days, and was then accompanied with vomiting. The patient did not believe she was with child, because the menses continued to flow, which had never been the case in her former pregnancies, nor did she experience the same feelings as before. On examining the body, it was found that the abdomen contained a large quantity of blood; the uterus was in a degree developed and it was larger on the right than on the left side. In the former part there was a rupture. The cavity of the viscus, lined by a shaggy membrane, did not communicate with the sac in which the fotus was. This cavity was formed in the substance of the uterus itself, and the foetus appeared to be about six weeks old. The internal orifice of the right fallopian tube was quite closed, whilst that of the left side was open.

A similar case has been described by $\mathrm{Mr}$. Hedrich ;* and Professor Carus of Dresden has given a drawing of the preparationt.

A peasant aged 35, the mother of three children, and who had likewise miscarried twice, thought herself again pregnant, because her menses had not

* Archiv. de Horn. Sept. and Oct. 1817.

+ See Dr. Carus' work already quoted. 
appeared for two months. Returning one evening from her work, she was seized with violent colic succeeded by vomiting, burning thirst, pallid countenance; shivering, and partial paralysis, alternately with spasms. These symptoms became rapidly worse, and the woman died the next day.

The abdomen was filled with blood, and the uterus increased in size. This organ, somewhat inflated, was empty, and its fundus was raised as high as the brim of the pelvis. On its right side, nothing was observed except an hydatid about the size of a nut. On the left side, there was a red prominence furnished with numerous blood vessels which gave it the appearance of an inflamed membrane; and upon this projection, there were two ruptures in the thinnest part of its parietes. Through these aperturesa probe passed into a sacciform cavity containing an embryo of from eleven to twelve weeks gestation enveloped as usual in the amnion and chorion; and upon these membranes the rudiments of the placenta were clearly distinguished. The sac inclosing the embryo was entirely separated from the proper cavity of the uterus, which organ was much larger than in the unimpregnated state, and lined by a spongy membrane, which was considered as a modification of membrana decidua. The orifice of the left fallopian tube was not to be detected by the most careful examination; it was indeed obliterated in its whole extent. 
M. T. F. Lobstein, in a report which he addressed to the faculty of medicine at Strasbourg relative to their anatomical museum, speaks of " a preparation in which a foetus is situated in the substance of the parietes of the uterus, without its being possible to account for its presence there." Being anxious to obtain a fuller detail of this preparation I requested $M$. Lobstein to examine it, and from his account it appears that it belonged in 1736 to the old university, and has been erroneously described in the catalogue. The embryo with its involucra was really in the cavity of the womb, and the placenta closely adhering to the membrana decidua was taken for the substance of the uterus, and for the lower and internal layer of it.* This case must not, therefore, be any longer considered as extra-uterine pregnancy of the nature I have been describingt.

Just as I was preparing this work for publication, I saw, in a pamphlet which has lately appeared, a case similar to those which form the subject of my essay. The author of the pamphlet evidently mistook the nature of the case. A girl aged 23, pregnant of her first child, well made, of a bilious temperament, and habitually silent, was admitted into the hospital on the 5th June, 1821. Her health

* Letter of M. T. F. Lobstein. Strasbourg, 1823.

+ Compte rendu à la Faculté de Médecine de Strasbourg, sur l'état actuel de son Musée anatomique, suivi du catalogue des objets qu'il renferme. Strasbourg, 1820. 
appeared very good until the $17 \mathrm{th}$, when she was attacked with slight vomiting. She appeared to be eight months advanced in pregnancy, and this corresponded with her own account. The cervix, however, preserved its usual length, and the orifice was not perceptibly dilated. On the $18 \mathrm{th}$, the vomiting was succeeded by faintings and a small frequent pulse. The abdomen, in which there was much shooting pain, appeared to contain something more than a gravid uterus, and conveyed to the hand the sensation of an obscure fluctuation. When the patient was interrogated as to the seat of the pain, she pointed to her neck and right shoulder. The symptoms soon became more urgent; the pulse beat feebly, a cold sweat spread over the body, and the countenance indicated extreme danger. The night passed in agony, and her death, preceded by delirium, took place early on the morning of the 19 th. The surgeon of the hospital, after ascertaining that delivery could not take place in the natural manner, proceeded to the Cæsarean operation. On opening the abdomen, he discovered a foetus in that cavity, with the head uppermost. Removing this fotus, together with the placenta, and numerous ciots of blood which covered the large intestines, he perceived an extensive rupture at the fundus of the uterus, which presented a remarkable appearance. This viscus, formed of two cavities, placed one above the other, and without any com. munication between them, bore the same relation as usual to the parietes of the pelvis, and the ab- 
dominal viscera. The upper division, containing the child, occupied the fundus of the uterus, and was triangular in shape. Its parietes, in the part corresponding to the rupture, were thin and membranous. The lips of the wound were fringed and dark coloured at some points; the lower part of this extraordinary sac was separated from the other by a partition half an inch thick. There was no trace of any communication between the two cavities. The broad ligament, the ovaries, and the tubes of the one side differed in their position from those of the other. On the right side they occupied the upper angle of this double uterus, which, externally viewed, had the appearance of a womb under common circumstances. On the left side, they were placed nearly in the centre of the left margin of the uterus, and were hidden in the cavity of the pelvis. A line drawn between these two points of insertion corresponded exactly with the division of the two cavities. The interior of the fœtal sac was precisely similar to that of the uterus after delivery. The placenta was inserted posteriorly and inferiorly in such a manner, that the partition contributed somewhat towards its formation. The proper cavity of the uterus, and likewise the vagina, were natural. The uterine cavity was separated, superiorly, by the partition already described from the fotal sac, and only communicated with the vagina. A sort of fatty membrane adhered feebly to its parietes. 
The foetus, which was a female, only weighed four pounds and a half; the umbilicus did not correspond to the middle of the body; the head was three inches and a quarter in diameter longitudinally, and two and a half inches transversely. The placenta was twenty-two inches in circumference, and three lines in thickness. The involucra ap. peared very thick.

The existence of this species of extra-uterine pregnancy being thus established, it will be interesting to investigate the cause and modes of its formation.

In the natural sciences, we must often confine ourselves to the mere observation of facts. It is easy to explain them when the mind is not scrupulous; but it is a proof of wisdom to attach little importance to explanations, and to suggest them only as speculations.

First Explanation.-It has been supposed that in this species of extra-uterine pregnancy, the two individual ova resulted from the same impregnation, and that the one had penetrated the other. There are more than twenty cases on record of foetus which have been developed in other fœtal bodies. In a late instance, the remains of a fotus were found in the scrotum and testicles of a new-born child; and I have often discovered such fragments in the abdomen of female children some months old. But it is impossible to establish any resem- 
blance between cases of this description, and those which form the subject of my Essay. In the former cases it is not so much a child as the remnants of a foetus which anatomists have discovered. Whilst, in the extra-uterine pregnancy of which we are treating, the embryo or foetus has been regularly developed, and its death has either preceded or followed that of the mother. Almost all these foetus have been found in adult women, who have borne several children; and not only the child, but the involucra and placenta were formed in the manner most favourable to the support of the fotal life.

Second Explanation.-Some who have not had the opportunity of examining these cases have supposed that the ovum might have passed along the fallopian tube upon the body of the uterus, under the peritoneum. This point is easily decided by dissection, as in examining the foetal pouch the peritoneal membrane may be easily distinguished from the substance of the uterus. These two structures could be easily séparated on the lips of the rupture, shewing that the external portion of the parietes of the sac were formed of peritoneum, and a layer of the substance of the uterus. The second explanation is, therefore, not more admissible than the first.

Third Explanation.-Can we account for the formation of two uterine cavities, and the pre- 
sence of a foetus in the upper one, by admitting the existence of a horizontal, incomplete partition dividing the uterus, and which in the progress of time becomes complete, and thus prevents all communication between the superior or fœtal, and the inferior or vaginal cavities?

We know of no instance of a uterus divided by a diaphragm, at right angles to the longitudinal axis of the organ. The horned wombs have a median partition, but it is always parallel to the longitudinal axis. If any aperture had existed, some trace of it would have been discovered, which we were unable to do.

Fourth Explanation.-It is possible to imagine that the lower part of the fallopian tube may have been obliterated, and that on the arrival of the ovum at the part thus obstructed, inflammation having been excited, it forced for itself a way into the substance of the uterus. The ovum would here be in a situation similar to a ball projected by gunpowder, penetrating the body, which is either carried to the surface or arrives at a visceral cavity by the process of an eliminating inflammation, which forms a way for it anteriorly, and closes the passage behind as it advances. The obliteration and thickening of the inferior part of the fallopian tube seem to give weight to this explanation. I would rather attribute the obliteration of the tube 
to inflammation preceding the descent of the ovum, than admit that this obliteration existed before impregnation.

A fifth explanation of this phenomenon may be found in a peculiar anatomical formation of the fallopian tube. It is known that at the inferior part next the uterus, there are to be observed on the inside of this canal the mouths of several vascular sinuses. Is it not possible that the ovum may have gradually dilated the orifice of one of these vessels, and, that, constantly impelled by the peristaltic force of the tube, the ovum may have formed a cavity for itself in the substance of the uterus towards its fundus, instead of passing into the cavity of the uterus. The fallopian tubes do not terminate at the external and upper part of the uterus, but penetrate into the substance of the uterus, as the ureters do into the bladder. If we admit the possibility of the case I have just stated, it will be seen that the ovum will have had a very short space to traverse in order to get into the substance of the uterus itself.

Sixth Explanation.-If we attempt to explain it on the ground of organic malconformation, analogy will supply us, in the instance of animals, with materials for another explanation.

In many of the mammalia the uterus is horned; that is, it is divided into two by a partition, and the 
body of the viscusis separated into two distinct cavities. The Monotremata, the Ornithorynchi, and the Didelphi are examples of this arrangement, which indeed may be seen to a certain degree in the uterus of the human fotus, and that of new born infants. I have repeatedly observed it myself, and M. Dumeril discovered in the body of a woman he lately opened, a double uterus, that is, the cavity of the viscus as well as the vagina were divided by a partition. Now, may it not be possible, that in such a uterus as this, when a fœtus enters one of the two cavities, it may distend it and communicate by its developement such motion to the partition that from longitudinal it may become transversal? My opinion is, that the explanation which supposes the ovum to enter one of the orifices of the uterine sinuses, is the most simple and admissible.

\section{All the facts related in this paper prove,}

1st. That extra-uterine pregnancies may occur in the substance of the uterus itself.

2dly. That in this as in all the other varieties of extra-uterine pregnancy, gestation arrives slowly and with difficulty at the ordinary period, and I think that in this variety, the ovum must experience more difficulty in its developement, than when it is in the fallopian tube.

3dly. That, at a period difficult to determine, a VOL. XIII. 
rupture takes place, accompanied with effusion of blood into the abdomen, and death is the quick and inevitable consequence.

4. That the membrana decidia exists in the uterus before the arrival of the germ, since in all my observation this membrane was distinctly formed although the tubes were obliterated.

5. That the membrana decidua does not belong to the embryo, properly so called, and that it is not indispensable to the nutrition of the foetus.

6th. That the uterus may be developed, and its cavity enlarged without the presence of a foetus in the usual place.

7th. That the placenta is not always formed in the human species, so as to constitute a uniform mass or cake, but that it may be developed at different distinct points of the ovum in the form of vascular penicilli as is seen in ruminating animals. The Solipeda for example. 


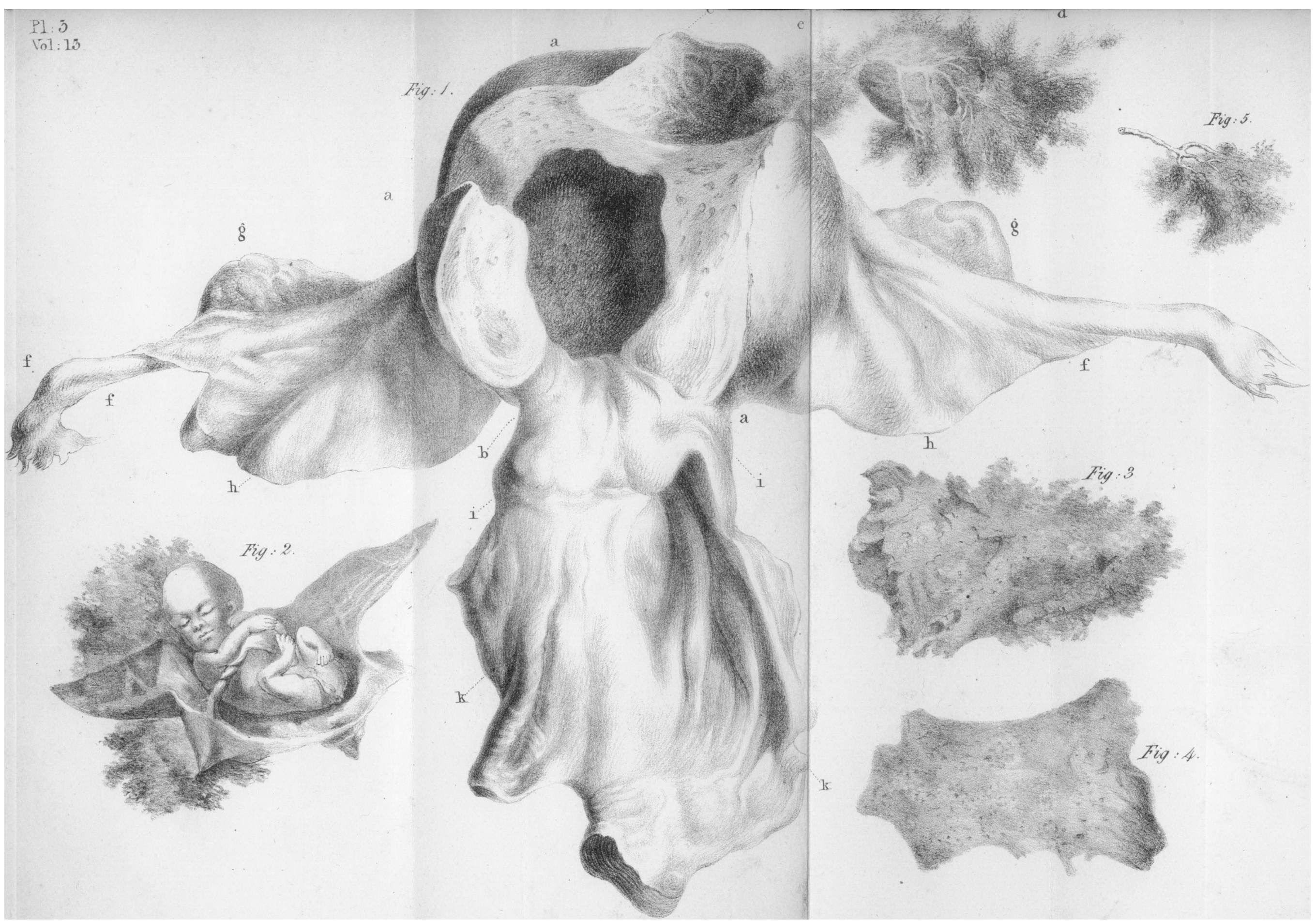

